\title{
Design and evaluation of electron beam energy degraders for breast boost irradiation
}

Jong In Park ${ }^{1,2,4}$, Sung Whan Ha ${ }^{2,3}$, Jung-in Kim ${ }^{3,5}$, Hyunseok Lee ${ }^{1,2}$, Jaegi Lee ${ }^{1,2}$, II Han Kim ${ }^{3,4}$ and Sung-Joon $\mathrm{Ye}^{1,2,3,4,5^{*}}$

\begin{abstract}
Background: For breast cancer patients who require electron boost energies between 6 and $9 \mathrm{MeV}$, an energy degraders (ED) in the $9 \mathrm{MeV}$ beamline was specially designed and manufactured to increase the skin dose of $6 \mathrm{MeV}$ and to reduce the penetration depth of $9 \mathrm{MeV}$ beams.

Methods: We used Monte Carlo (MC) techniques as a guide in the design of ED for use with linear accelerators. In order to satisfy percent depth dose (PDD) characteristics and dose profile uniformity in water, the shape and thickness of Lucite ${ }^{\oplus D}$ in the $9 \mathrm{MeV}$ beamline was iteratively optimized and then manufactured. The ED geometry consists of a truncated cone attached on top of a plane plate, with total central thickness of $1.0 \mathrm{~cm}$. The ED was placed on the lower most scraper of the electron applicator. The PDDs, profiles, and output factors were measured in water to validate the MC-based design.
\end{abstract}

Results: Skin doses with the EDs increased by 8-9\%, compared to those of the $9 \mathrm{MeV}$ beam. The outputs with the EDs were 0.882 and 0.972 for $10 \times 10$ and $15 \times 15 \mathrm{~cm}^{2}$ cones, respectively, as compared to that of a conventional $9 \mathrm{MeV}$ beam for a $10 \times 10 \mathrm{~cm}^{2}$ cone. The X-ray contamination remained less than $1.5 \%$. In-vivo measurements were also performed for three breast boost patients and showed close agreement with expected values.

Conclusions: The optimally designed ED in the $9 \mathrm{MeV}$ beamline provides breast conserving patients with a new energy option of $7 \mathrm{MeV}$ for boost of the shallow tumor bed. It would be an alternative to bolus and thus eliminate inconvenience and concern about the daily variation of bolus setup.

Keywords: Electron energy degrader, Breast boost irradiation, Monte Carlo simulation, Linear accelerator

Abbreviations: ED, Energy degrader; PDD, Percent depth dose; PTV, Planning target volume; OAR, Organ at risk; LINAC, Linear accelerator; eMLC, Electron multi-leaf collimator; FLEC, The few leaf electron collimator; MC, Monte Carlo; SSD, Source to surface distance; FWHM, Full width at half maximum; PMMA, Polymethly methacrylate; TPS, Treatment planning system; $d_{\max }$ Depth of dose maximum; $R_{90}, 90 \%$ of dose maximum; $R_{80}, 80 \%$ of dose maximum; $R_{50}, 50 \%$ of dose maximum; $E_{0}$, The most probable energy of the incident electron beam; $X_{\text {con, }}, X_{\text {-ray }}$ contamination that is extracted from $R_{\mathrm{p}}$ plus $2 \mathrm{~cm}$; $T_{\mathrm{t}}$, Top layer thickness; $T_{\mathrm{b}}$, Bottom layer thickness; $r$, The radius of the top layer; EP-P, A simple plate design of ED; A/P, Area to perimeter

\footnotetext{
* Correspondence: sye@snu.ac.kr

${ }^{1}$ Department of Transdisciplinary Studies, Program in Biomedical Radiation

Sciences, Seoul National University Graduate School of Convergence Science

and Technology, Seoul 151-742, Korea

${ }^{2}$ Institute of Radiation Medicine, Seoul National University Medical Research

Center, Seoul, Korea

Full list of author information is available at the end of the article
} 


\section{Background}

Electron boost irradiation for breast cancer patients is routinely practiced in modern radiotherapy clinics. Electron beams are capable of covering planning target volumes (PTV) with an appropriate prescription dose while sparing the underlying critical structures [1]. Electron beams are advantageous over photon beams for breast boost irradiation due to more effective sparing of distally located organs at risk (OAR), and the ability to deliver a fairly uniform dose to the target volume [2]. In general a depth of $90 \%$ or $80 \%$ of dose maximum is the therapeutic range of electron beam [1]. Thus, in order to allow personalized treatments with an optimal energy, fine energy spacing of electron beams of commercial linear accelerators (LINAC) is necessary. However, with an electron beam of 6 or $9 \mathrm{MeV}$, it is difficult for the skin dose to reach $90 \%$ of the dose maximum while at the same time having the therapeutic range located between the distal end of the target volume and the proximal part of the OAR.

In order to increase the skin dose while retaining high dose fall-off beyond the depth of dose maximum $\left(d_{\max }\right)$, a thin tantalum wire mesh placed on the patient's skin has been proposed $[3,4]$. A similar energy control has also been achieved through the use of a high-density metal foil bolus [5]. A water equivalent bolus has been widely used during part of the treatment to raise the skin dose and to reduce the energy or therapeutic range $[6,7]$. However, daily setup variation with the bolus may cause uncertainties in the dose delivered to the target volume.

An advanced technique using a prototype electron multi-leaf collimator (eMLC) to create narrow and segmented beams has been used to modulate electron energy without the setup variation [8-15]. Modulation of adjacent narrow segments with the eMLC enhances the skin dose while sparing surrounding normal tissues [16]. In addition to the eMLC, the few leaf electron collimator (FLEC) has also been used for an alternative to bolus for boost treatment of tumor bed in breast cancer [17, 18]. Although this technique is promising, it requires an add-on to the LINAC (i.e., the eMLC) and is still a kind of prototype. Options for energy spacing remain restricted in clinical situations.

It was well known that a spoiler made of low-atomic number $(\mathrm{Z})$ material could enhance the skin dose for electron treatments with beam energies between 6 and $12 \mathrm{MeV}$ while limiting the penetration depths above OARs [19]. In this study we produced an electron beam spoiler without modifying components of the LINAC. The spoiler can provide a therapeutic range between 6 and $9 \mathrm{MeV}$, and is thus termed an energy degrader (ED). In addition, it could eliminate concern about the daily variation of bolus setup. We performed Monte Carlo
(MC) simulations to optimize the design of the ED system by calculating beam characteristics such as depth doses, uniformity, dose rates, and bremsstrahlung contamination. Prior to clinical use, rigorous measurements were taken to validate the MC-based optimization.

\section{Methods \\ Clinical linear accelerator}

The medical LINAC used in this study was Varian Trilogy (Varian Oncology Systems, Palo Alto, CA). Six electron energies $(4,6,9,12,16$ and $20 \mathrm{MeV})$ were available, and fields were shaped with open walled applicators consisting of three scrapers and matched scattering foils. A final field-defining Cerrobend cutout was placed on the lowermost scraper. The electron applicators have a nominal source-to-end of applicator distance of $95 \mathrm{~cm}$. This indicates a $5 \mathrm{~cm}$ air-gap between the applicator end and the standard source-to-surface distance $(\mathrm{SSD})=100 \mathrm{~cm}$ plane. The beams investigated in this study were the standard $9 \mathrm{MeV}$ beam and an ED-moderated beam in the standard $9 \mathrm{MeV}$ beamline, hereafter often denoted $7 \mathrm{MeV}$ beam since an ED of $1 \mathrm{~cm}$ water-equivalent thickness moderates electron energy by $2 \mathrm{MeV}$ approximately. They were collimated by $10 \times 10$ and $15 \times$ $15 \mathrm{~cm}^{2}$ applicators.

\section{Monte Carlo simulation}

The geometry and compositions of the primary collimator, vacuum window, scattering foil, monitoring ionchamber, mirror, movable jaws, etc. for the $9 \mathrm{MeV}$ electron mode, and the $10 \times 10$ and $15 \times 15 \mathrm{~cm}^{2}$ applicators and their scrapers were obtained from information supplied by the manufacturer. The construction details of the LINAC treatment head were also provided by the manufacturer, whereas the energy degraders were built in our laboratory. The uppermost and middle scrapers of the electron applicator were modeled using the EGSnrc/BEAMnrc component module (CM) APPLICAT [20]. The lowermost scraper was modeled by PYRAMIDS to insert the ED into the cutout insert, and the ED was modeled by CONESTAK. Our EGSnrc/ BEAMnrc simulations consisted of two major steps. The first step involved adjusting the primary electron beam parameters of our LINAC to match the $9 \mathrm{MeV}$ beam data measured. In the second step, these beam parameters were then used to compute dose distributions with various different designs of energy degraders, and thereby provided guidance for the manufacturing of optimized EDs. The EGSnrc/DOSXYZnrc code was used to calculate dose distributions in a $30 \times 30 \times 30 \mathrm{~cm}^{3}$ water phantom at $100 \mathrm{~cm} \mathrm{SSD}$, irradiated by the $99.9 \mathrm{~cm}$ SSD phase space determined in the previous simulations [21]. The doses in the water phantom were scored in voxels of $0.5 \mathrm{~cm}$ (width) $\times 0.5 \mathrm{~cm}$ (length) $\times 0.2 \mathrm{~cm}$ 

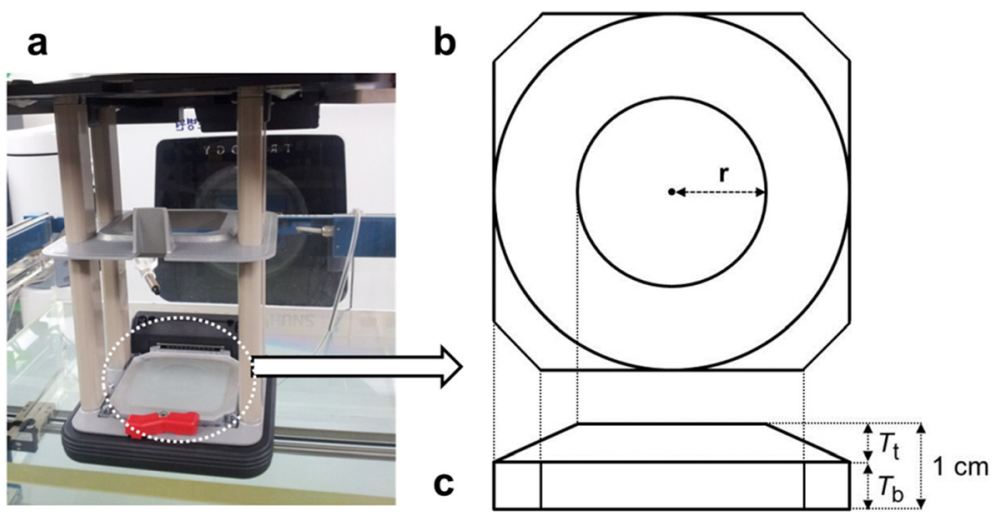

Fig. 1 Construction of energy degrader. Construction of the energy degrader (ED) for $7 \mathrm{MeV}$ beam investigated using the Monte Carlo simulations. The shape of ED was a truncated cone attached on the plane plate and the central thickness of ED was $1.0 \mathrm{~cm}$. a A picture of the optimized energy degrader placed on the plane plate of the lowermost scraper for $10 \times 10 \mathrm{~cm}^{2}$ cone. It was secured in the cutout insert. $\mathbf{b}$ The top view of ED; the parameter $r$ is the radius of circle on the upper layer. $\mathbf{c}$ The side view of ED; $T_{t}$ is the thickness for upper layer and $T_{b}$ is the thickness of bottom layer. Ratio of two layer's thickness varied with design-to-design

(depth). In the EGSnrc/BEAMnrc simulations, the initial number of histories was $3.0 \times 10^{8}$ particles emitting from the vacuum window. Approximately $1.0 \times 10^{8}$ particles were scored in the phase space at $99.9 \mathrm{~cm} \mathrm{SSD}$ with an air slab of $0.1 \mathrm{~cm}$ thickness. In the EGSnrc/DOSXYZnrc simulations, $3.0 \times 10^{8}$ particles were sampled from the $99.9 \mathrm{~cm} \mathrm{SSD}$ phase space file as a source, yielding statistical uncertainties of $< \pm 2 \%$ along the cross-beam profiles of -8 to $+8 \mathrm{~cm}$ for a $10 \times 10 \mathrm{~cm}^{2}$ field, and along the cross-beam profiles of $-9 \mathrm{~cm}$ to $+9 \mathrm{~cm}$ for $15 \times$ $15 \mathrm{~cm}^{2}$. Such a number of particles required the phase space file to be recycled 1 up to three times. Statistical uncertainties in depth doses along the central axis were less than $\pm 1 \%$ except in the bremsstrahlung tails $(< \pm 2.5 \%)$. In accordance with previously published papers [20, 22-28], ECUT (the energy cutoff for electron transport) was set to $700 \mathrm{keV}$ for the EGSnrc/BEAMnrc and EGSnrc/DOSXYZnrc simulations. PCUT (the energy cut-off photon transport) was set to $10 \mathrm{keV}$ for both simulations. Below these cut-off energies the kinetic energy of the particle was considered to be absorbed locally.

To find the parameters of the electron beam incident on the vacuum window, we followed published procedures by matching our calculated depth doses and crossbeam profiles to our measurements in a water phantom $[22,29,30]$. We started with published beam parameters from a model of a linear accelerator identical to ours, and made small fine-tuning adjustments until the best match was found [22, 23, 28, 31]. Measured cross-beam dose profiles were found to be symmetric and we thus used a normally incident beam with no lateral shift. Varied parameters of the parallel circular beam included the full width at half maximum (FWHM) of the Gaussian radial distribution and the energy of the incident electron source. Tuning of incident electron parameters was performed by comparing calculated and measured relative central axis depth doses and cross-beam profiles at $100 \mathrm{~cm} \mathrm{SSD} \mathrm{for} 10 \times 10$ and $15 \times 15 \mathrm{~cm}^{2}$ ones.

We investigated beam parameters with incident energies of 9.75, 9.85, 9.95 and $10.05 \mathrm{MeV}$ having Gaussian radial distributions with FWHM of $0.12,0.13,0.15 \mathrm{~cm}$. The beam parameters that yielded the closest agreement between simulations and measurements were considered as the best estimate of the actual beam parameters and used for all subsequent calculations.

\section{Design of the energy degrader}

It is possible to change the energy of the electron beam by tuning the current of the bending magnet from the standard 6 or $9 \mathrm{MeV}$ [32]. However, this procedure requires the standard energy to be replaced with a different energy. In contrast, we chose to insert an ED into an

Table 1 Parameterized values to optimize the energy degrader. Parameterized values to optimize the energy degrader for $10 \times$ $10 \mathrm{~cm}^{2}$ and $15 \times 15 \mathrm{~cm}^{2}$ cone size. $r$ is the radius of top layer. $T_{\mathrm{t}}$ is the top layer thickness. $T_{\mathrm{b}}$ is the bottom layer thickness. $T_{\mathrm{t}}+$ $T_{\mathrm{b}}$ is equal to $1.0 \mathrm{~cm}$

\begin{tabular}{|c|c|c|c|c|c|c|c|}
\hline \multicolumn{4}{|c|}{$10 \times 10 \mathrm{~cm}^{2}$ cone } & \multicolumn{4}{|c|}{$15 \times 15 \mathrm{~cm}^{2}$ cone } \\
\hline & $r$ & $T_{\mathrm{t}}$ & $T_{\mathrm{b}}$ & & $r$ & $T_{\mathrm{t}}$ & $T_{\mathrm{b}}$ \\
\hline$\overline{E D}-P^{a}$ & - & - & 1.0 & $E D-P^{a}$ & - & - & 1.0 \\
\hline ED-1 & 2 & 0.7 & 0.3 & ED-1 & 2 & 0.6 & 0.4 \\
\hline ED-2 & 2 & 0.6 & 0.4 & ED-2 & 3 & 0.6 & 0.4 \\
\hline ED-3 & 2 & 0.5 & 0.5 & ED-3 & 6 & ' & 0.4 \\
\hline ED-4 & 2 & 0.4 & 0.6 & ED-4 & 4 & 0.3 & 0.7 \\
\hline ED-5 & 2 & 0.3 & 0.7 & ED-5 & 6 & 0.3 & 0.7 \\
\hline
\end{tabular}

${ }^{\mathrm{a} E D-P}$ is a Lucite ${ }^{\oplus}$ slab plate, thickness of which is $1.0 \mathrm{~cm}$ Highlighted bold ED-4 was selected as an optimal design 


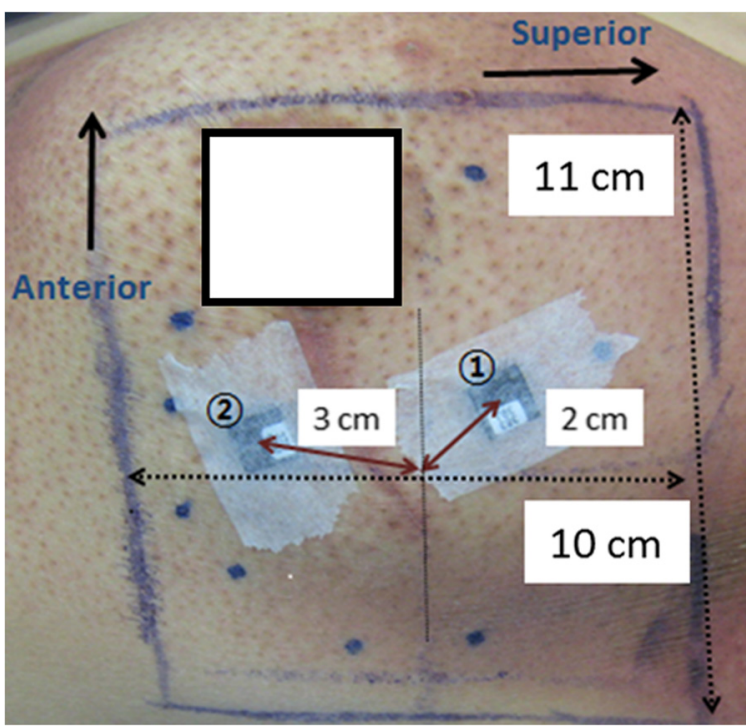

Fig. 2 The setup picture of in-vivo dosimetry for patient 2. NanoDot ${ }^{\text {TM }}$ optically stimulated luminescent (OSLD) dosimeters (Landauer Inc., Glenwood, IL) were used to measure the irradiated dose. The $15 \times$ $15 \mathrm{~cm}^{2}$ cone was used to irradiate the target which size was $10 \mathrm{~cm}$ (superior-to-inferior) $\times 11 \mathrm{~cm}$ (Anterior-to-inferior). OSLD3 and OSLD4 are numbered 1 and 2 in the figure. OSLD3 and OSLD4 were located at the $2 \mathrm{~cm}$ and $3 \mathrm{~cm}$ from the isocenter, respectively electron applicator. Once the source parameters of the $9 \mathrm{MeV}$ beam were determined, various EDs inserted into an applicator of either $10 \times 10$ or $15 \times 15 \mathrm{~cm}^{2}$ cone were tested using $\mathrm{MC}$ simulations. We limited our investigation to the EDs placed on the lowermost scraper, which was the case to minimize scattered doses outside of treatment field (Fig. 1a). With an ED in place, dose uniformity at depths could be worse than that of electron beams with no ED (ED-P). Thus one of our design goals was to achieve a uniform dose region at treatment depths. A simple double-layer approach was chosen for the ED design as shown in Fig. 1b-c. Our investigations included six different ED designs for both $10 \times 10$ and $15 \times 15 \mathrm{~cm}^{2}$ cones, as summarized in Table 1 . Five of the ED designs incorporated a truncated cone attached on top of the plane plate, where the bottom layer was a plate shape and the edge of the upper layer was carved to improve uniformity as shown in Fig. 1c. As shown in Table 1 , the radius of the top layer $r$, top layer thickness $T_{\mathrm{t}}$, and bottom layer thickness $T_{\mathrm{b}}$ were chosen to achieve the best possible uniformity at $d_{\max }$ of the cross-beam dose profile. The total thickness of the Lucite $^{\circ}\left(T_{\mathrm{t}}+T_{\mathrm{b}}\right)$ was $1 \mathrm{~cm}$ to reduce electron energy by approximately by $2 \mathrm{MeV}$. The radius of the top plate was fixed as an empirically chosen value $(2 \mathrm{~cm})$ when simulating the $10 \times 10 \mathrm{~cm}^{2}$ field because varying
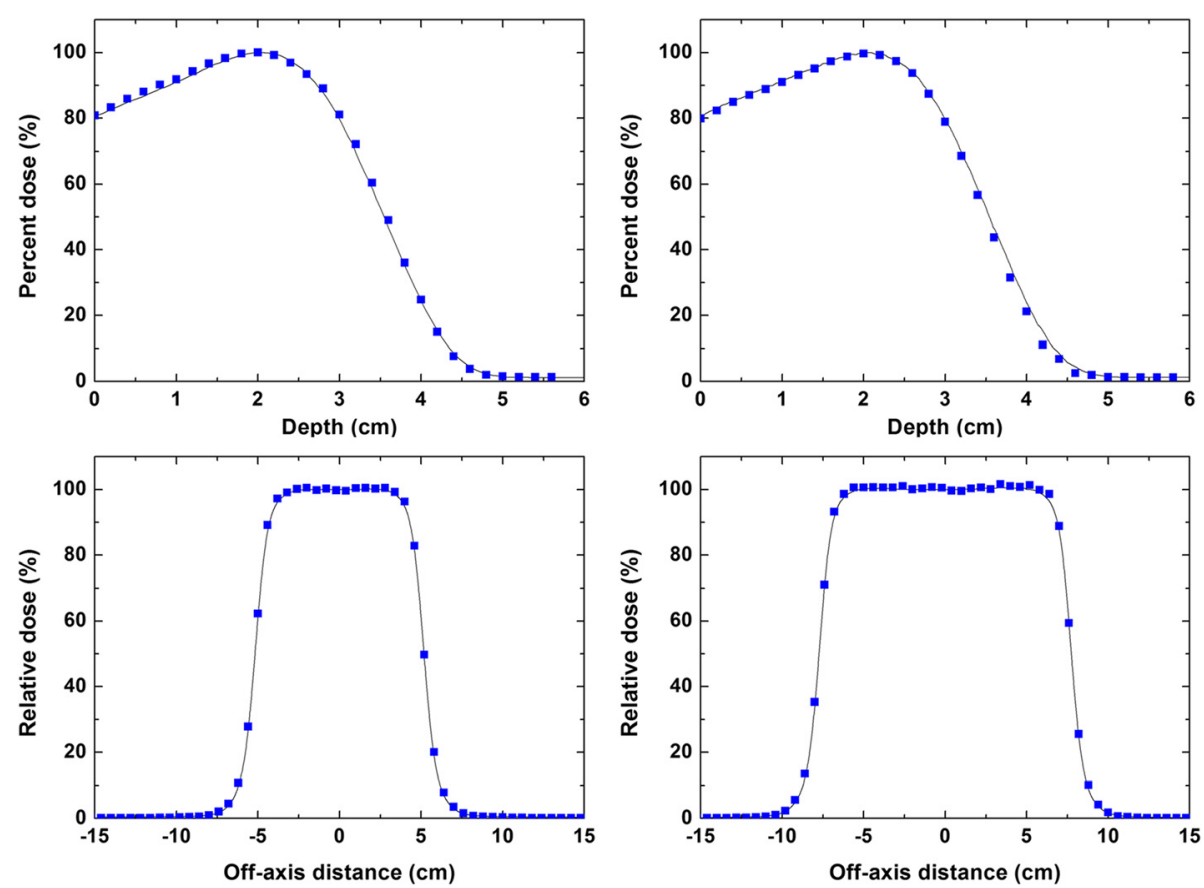

Fig. 3 Comparison of the measured and calculated beam characteristics curves for $9 \mathrm{MeV}$ beam. A comparison of the measured and calculated relative central-axis depth dose curves in water for $9 \mathrm{MeV}$ beam of (a) $10 \times 10 \mathrm{~cm}^{2}$ and (b) $15 \times 15 \mathrm{~cm}^{2}$ cones. Measured and calculated crossbeam profiles at the depth of dose maximum in water for $9 \mathrm{MeV}$ electron beam of (c) $10 \times 10 \mathrm{~cm}^{2}$ and (d) $15 \times 15 \mathrm{~cm}^{2}$ cones. Solid lines indicate measurements data with an ionization chamber (CC13). Squares indicate the calculated data from Monte Carlo simulations. The energy and lateral spread of incident electron beam for MC simulations were $9.85 \mathrm{MeV}$ and $0.13 \mathrm{~cm}$ FWHM of Gaussian distribution, respectively 
only $T_{t}$ or $T_{b}$ was sufficient for improving the profile uniformity. In addition, a simple plate (ED-P) was simulated for comparison. Output factors were calculated to quantify the output loss by scattering with the EDs and compared with measured values. The reference configuration of the output factor was a $10 \times 10 \mathrm{~cm}^{2}$ open applicator field with the water phantom at a nominal SSD of $100 \mathrm{~cm}$. The output factor for a given field with the ED was calculated by taking the ratio of the maximum calculated dose in that configuration to the maximum calculated dose in the reference configuration. Output factors with optimized EDs were calculated for $10 \times 10 \mathrm{~cm}^{2}$ and $15 \times$ $15 \mathrm{~cm}^{2}$ applicators.

Lucite, also known as polymethly methacrylate (PMMA), was chosen as the selected material for the ED. Lucite ${ }^{\circ}$ is a water-equivalent material with a relatively low Z, which is expected to decrease electron energy by approximately $2 \mathrm{MeV}$ per $\mathrm{cm}$ and to cause relatively low X-ray contamination [33].

\section{Measurement with ion-chamber}

Relative central-axis depth dose and cross-beam dose profiles for the standard $9 \mathrm{MeV}$ electron beam were measured in water using a $\mathrm{CC} 13$ thimble ion-chamber (PTW, Freiburg, Germany) in a Blue phantom (IBA dosimetry, Louvain-la-Neuve, Belgium) at $100 \mathrm{~cm}$ SSD. These measured data were acquired and analysed with data acquisition software (OmniPro Accept version 7.1A). The electron beams were perpendicular to the phantom within the accuracy of machine setup. Steps of $0.5 \mathrm{~mm}$ with a $1 \mathrm{~s}$ dwell time at each measurement position were used. The depth of dose maximum, along with 90,80 and $50 \%$ of dose maximum are represented by $d_{\max }, R_{90}, R_{80}$, and $R_{50}$. The practical range, which is the depth where the tangent to the linear portion of the central-axis depth-dose curve intersects the extrapolated $\mathrm{X}$-ray contamination, is represented by $R_{\mathrm{p}}$. The most probable energy $\left(E_{0}\right)$ of the incident electron beam was calculated. All measurements and corrections in this study were done in accordance with the recommendations of the AAPM TG-51 protocol and TG-25 report [34, 35].

Output factors were measured with a $\mathrm{CC} 13$ ionchamber by placing the effective point of measurement at the predetermined measured position of $d_{\max }$ for the given and reference fields. The TG-51 protocol recommends the use of a parallel plate chamber for electron beams with $R_{50}$ less than or equal to $4.3 \mathrm{~cm}$ [34]. The thimble ionization chamber (Scanditronix Medical AB, Uppsala, Sweden) was used to measure the output factors for electron beams of energy less than $10 \mathrm{MeV}$ [36-38]. The thimble ionization chamber has the geometry
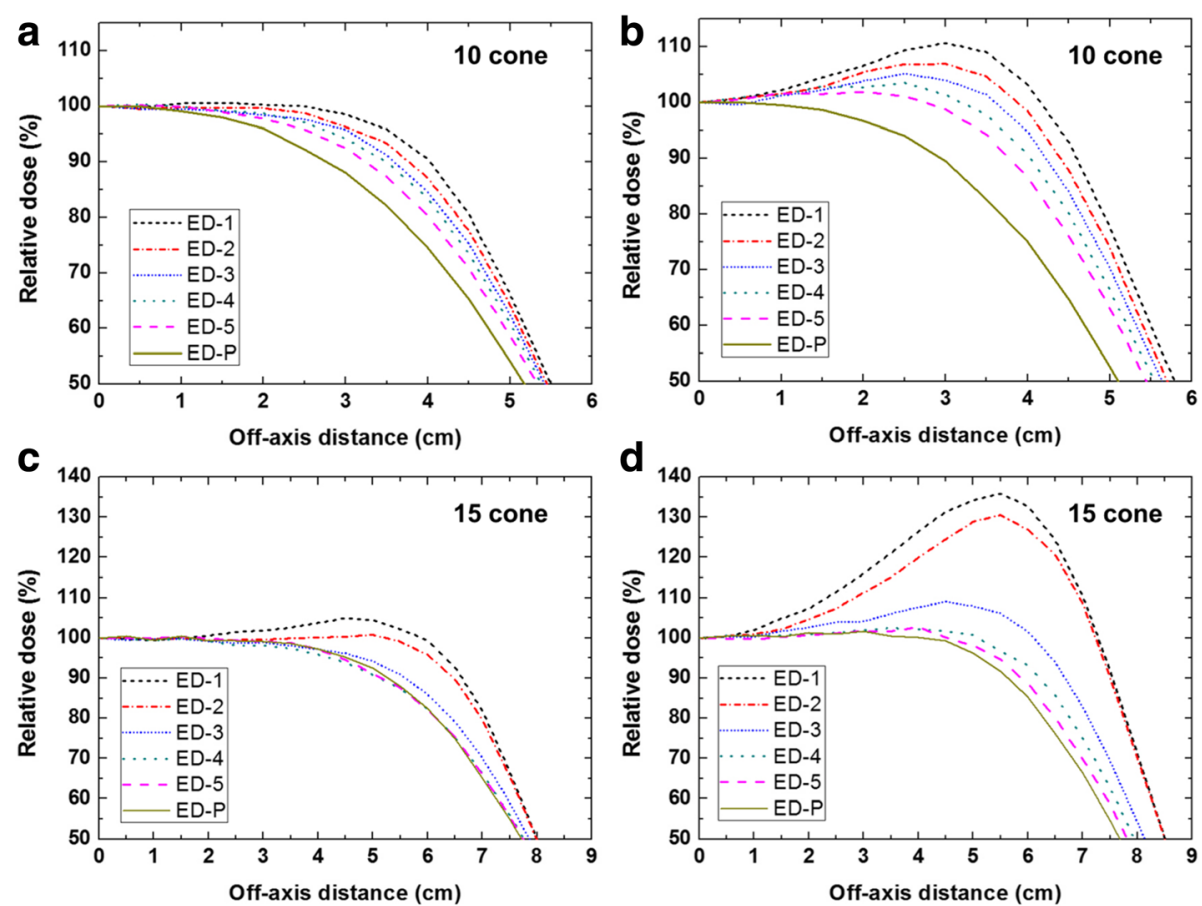

Fig. $4 \mathrm{MC}$ beam profiles of the $7 \mathrm{MeV}$ electron beams for six designs. MC beam profiles of $10 \times 10 \mathrm{~cm}^{2}$ cone were obtained (a) $d_{\max }$ and (b) $R_{50}$ for six designs of the energy degrader (ED). MC beam profiles of $15 \times 15 \mathrm{~cm}^{2}$ cone were obtained at (c) $d_{\max }$ and (d) $R_{50}$ for six designs of the ED. An optimal design for $7 \mathrm{MeV}$ beam was selected by considering uniformity of profile at $d_{\max }$ and $R_{50}$. ED-4 was the final design for $10 \times 10 \mathrm{~cm}^{2}$ and $15 \times 15 \mathrm{~cm}^{2}$ cones 

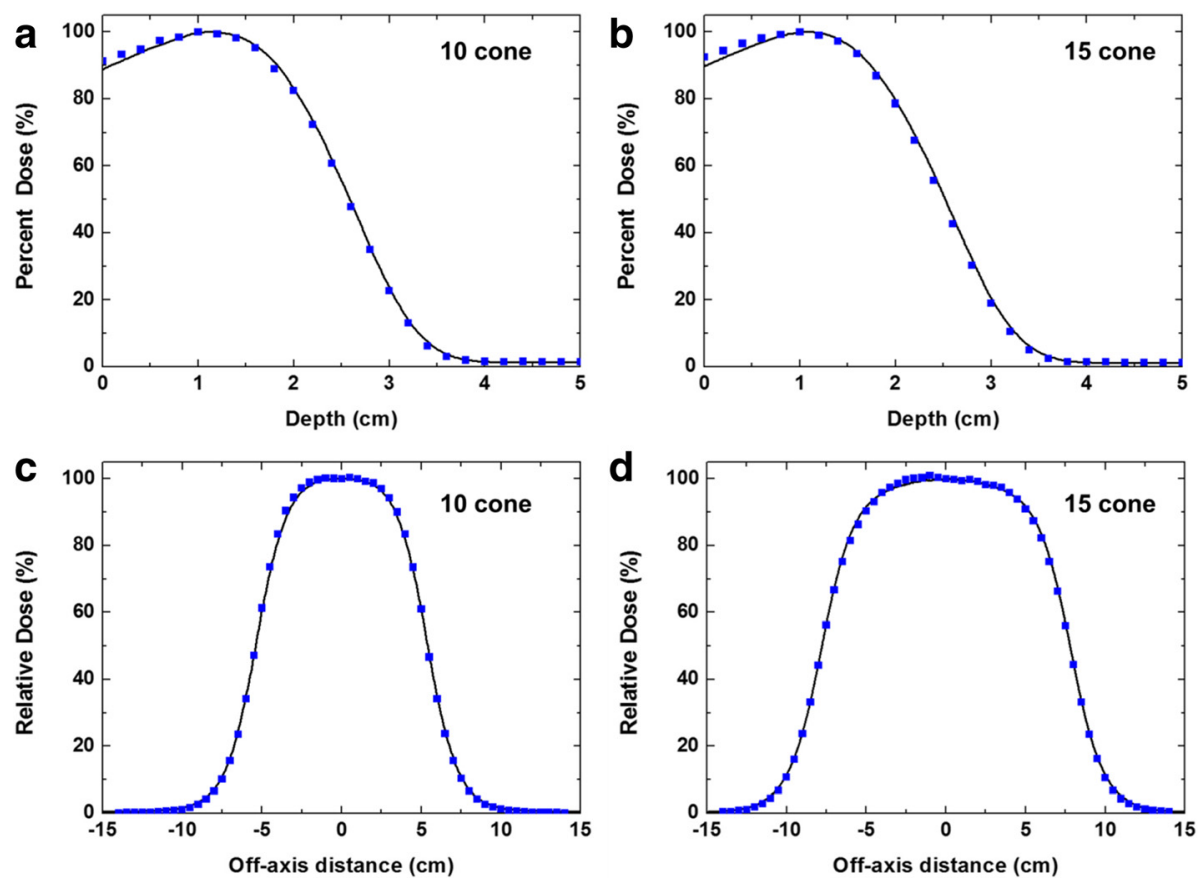

Fig. 5 Comparison of the measured and calculated beam characteristics for new $7 \mathrm{MeV}$ beam. A comparison of the measured and calculated relative central-axis depth dose curves in water for $7 \mathrm{MeV}$ beam of (a) $10 \times 10 \mathrm{~cm}^{2}$ and (b) $15 \times 15 \mathrm{~cm}^{2}$ cones. Measured and calculated crossbeam profiles at the depth of dose maximum in water for $7 \mathrm{MeV}$ electron beam of (c) $10 \times 10 \mathrm{~cm}^{2}$ and (d) $15 \times 15 \mathrm{~cm}^{2}$ cones. Solid lines indicate measurements data with an ionization chamber (CC13). Squares indicate the calculated data from Monte Carlo simulations

almost identical to a CC13 ion-chamber. Several other studies also used the cylindrical chambers to measure dosimetry data for low energy electron beams [32, 39]. The reference field for output factor measurements was an open field with the $10 \times 10 \mathrm{~cm}^{2}$ applicator. In this study, a
$10 \times 10 \mathrm{~cm}^{2}$ applicator defines a field of $10 \times 10 \mathrm{~cm}^{2}$ at $\mathrm{SSD}=100 \mathrm{~cm}$. The diameters of the Cerrobend cutouts were $3,4,5,6,7,8$ and $9 \mathrm{~cm}$ for the $10 \times 10 \mathrm{~cm}^{2}$ cone, and $8,9,10$ and $12 \mathrm{~cm}$ for $15 \times 15 \mathrm{~cm}^{2}$. Values of cutout factor were listed along the ratio of area to

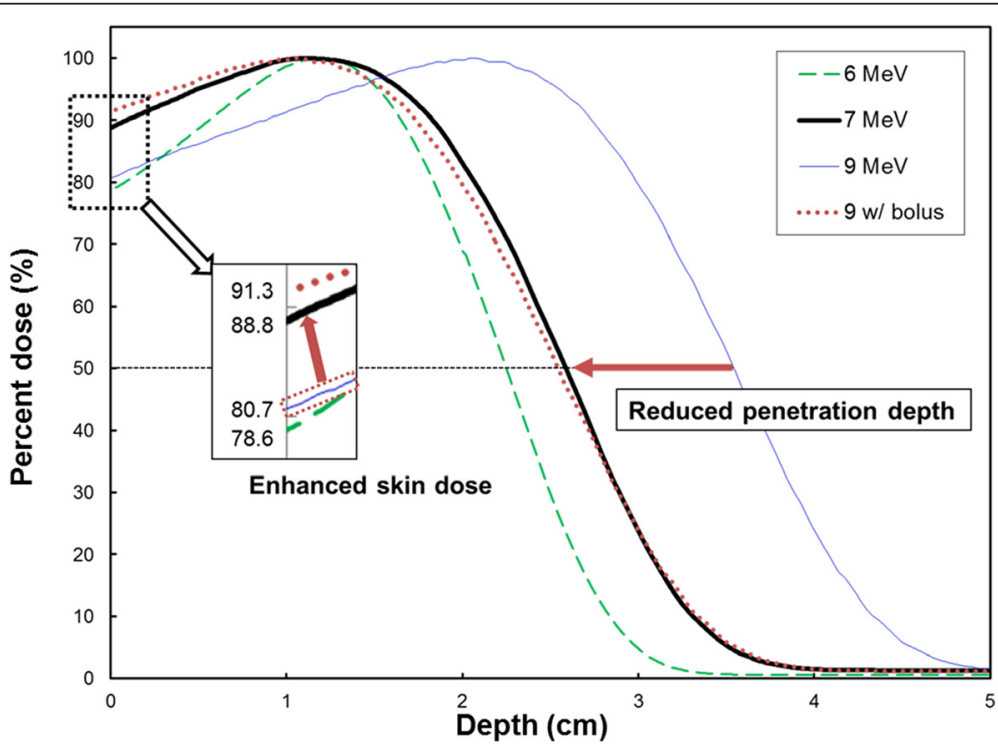

Fig. 6 Comparison of measured depth-dose curves of electron beams. Comparison of measured depth-dose curves for new $7 \mathrm{MeV}$ (bold solid), $9 \mathrm{MeV}$ (solid), $6 \mathrm{MeV}$ (dashed) and $9 \mathrm{MeV}$ with $1 \mathrm{~cm}$ bolus (dotted) for $10 \times 10 \mathrm{~cm}^{2}$ cone. Reduced penetration depth and enhanced skin doses for $7 \mathrm{MeV}$ beam were shown in this figure 
Table 2 Central-axis depth dose characteristics for $10 \times 10 \mathrm{~cm}^{2}$ cone. Central-axis depth dose characteristics for $7 \mathrm{MeV}, 9 \mathrm{MeV}$, and $9 \mathrm{MeV}$ with $1 \mathrm{~cm}$ bolus of $10 \times 10 \mathrm{~cm}^{2}$ cone. $X_{\text {con }}$ indicates $X$-ray contamination that is extracted from $R_{\mathrm{p}}$ plus $2 \mathrm{~cm}$

\begin{tabular}{lcccccccc}
\hline Nominal energy $(\mathrm{MeV})$ & Surface dose $(\%)$ & $d_{\max }(\mathrm{cm})$ & $R_{90}(\mathrm{~cm})$ & $R_{80}(\mathrm{~cm})$ & $R_{50}(\mathrm{~cm})$ & $R_{\mathrm{p}}(\mathrm{cm})$ & $\mathrm{E}_{0}(\mathrm{MeV})$ & $X_{\text {con }}(\%)$ \\
\hline 7 & 88.8 & 1.16 & 1.83 & 2.07 & 2.59 & 3.35 & 6.03 & 1.3 \\
9 & 80.7 & 2.05 & 2.74 & 2.99 & 3.54 & 4.39 & 8.25 & 1.2 \\
9 with bolus & 91.3 & 1.05 & 1.74 & 1.99 & 2.54 & 3.33 & 5.92 & - \\
\hline
\end{tabular}

perimeter $(\mathrm{A} / \mathrm{P})$ of the equivalent square field for a circular cutout (Table 5).

\section{In-vivo dosimetry}

The new $7 \mathrm{MeV}$ beam was implemented to our in-house MU calculation software. Dose characteristics were investigated from $\mathrm{SSD}=90 \mathrm{~cm}$ to $110 \mathrm{~cm}$. This software was based on AAPM TG-71 protocol [40]. The reference dosimetry following the AAPM TG-51 protocol was performed to calibrate the OSLDs [34, 41]. The PTW30013 Farmer type chamber (PTW, Freiburg, Germany) was used for this purpose. It was reported that the cylindrical PTW Farmer chamber and the parallel plate PTW Roos chamber agreed within $1 \%$ for the 4 and $6 \mathrm{MeV}$ energies, and within $0.5 \%$ for the 9 and $12 \mathrm{MeV}$ energies [42].

Calibrated OSLDs were in agreement within $2 \%$ with dose readings of an ion-chamber at $d_{\max }$ of the $7 \mathrm{MeV}$. To minimize statistical uncertainty, five dose readings were performed and averaged over each dosimeter. Invivo dosimetry was performed for the first three patients who were treated with the newly-developed $7 \mathrm{MeV}$ beam. The setup of the OSLD was shown in Fig. 2 (patent 2). The treatment field was defined by a radiation oncologist on the basis of clinical examination. Monitor units were calculated using the in-house calculation software. Daily 180 cGy fractions were prescribed at $d_{\max }$. Two OSLDs were placed on the skin of each patient.

\section{Results}

Head component modelling for source parameters

As shown in Fig. 3 , the calculated values of $d_{\text {max }}, R_{50}$, and $R_{p}$ agreed well with measured data, within $1 \mathrm{~mm}$, when incident electron energy of $9.85 \mathrm{MeV}$ was chosen. The calculated cross-beam dose profiles at $d_{\text {max }}$ and $R_{50}$ agreed well with measured data, within $2 \%$, except in penumbra regions when $0.13 \mathrm{~cm}$ FWHM of the incident electron beam was chosen. Thus, the energy and lateral spread of the incident electron beam for the standard
$9 \mathrm{MeV}$ electron beam were determined to be $9.85 \mathrm{MeV}$ and $0.13 \mathrm{~cm}$ FWHM of Gaussian distribution, respectively. All subsequent simulations with the various energy degraders were carried out using these two source parameters.

\section{Monte Carlo simulations of the energy degraders}

Calculated relative depth-dose curves along the centralaxis were almost identical among the all ED designs described in Table 1. Therefore, dose profiles at $d_{\text {max }}$ and $R_{50}$ with the EDs were mainly used to evaluate the performance of the EDs. Figure 4 shows calculated crossbeam dose profiles along the $\mathrm{x}$-axis normalized to the value at the central-axis. As shown in Fig. 4, the crossbeam dose profile of ED-P falls off rapidly as distance from the central axis increases. The ED-P failed to achieve acceptable profile uniformity.

For the $10 \times 10 \mathrm{~cm}^{2}$ cone (Fig. 4a and b), electrons penetrating through the thick part of the ED degrade their energy more than those penetrating the thinner regions. Thus, truncated EDs show better uniformity with increasing $T_{t}$ or decreasing $T_{b}$, as compared to ED-P. Considering uniformity and the horn in the dose profiles at $d_{\max }$ and $R_{50}$, ED-4 was selected as the optimal design for the $7 \mathrm{MeV}$ beam of $10 \times 10 \mathrm{~cm}^{2}$ cone. Fig. $4 \mathrm{c}$ and $\mathrm{d}$ for the $15 \times 15 \mathrm{~cm}^{2}$ cone show the effects of the radius of the top layer $(r)$ on the profile at $d_{\max }$ and $R_{50}$, respectively. The horn dose at $R_{50}$ increased as $r$ decreased as shown in Fig. 3d. ED-1, ED-2 and ED-3 show unacceptable dose horns at $4-7 \mathrm{~cm}(>10 \%)$. As ED-4 showed less penumbra region at $R_{50}$ than did ED-5, ED4 was selected as an optimized design for our $7 \mathrm{MeV}$ beam of $15 \times 15 \mathrm{~cm}^{2}$ cone.

\section{Relative central-axis depth-dose curve and cross-beam dose profiles}

Based on the guidance of the previous section, the two optimized EDs for $10 \times 10 \mathrm{~cm}^{2}$ and $15 \times 15 \mathrm{~cm}^{2}$ cones

Table 3 Central-axis depth dose characteristics for $15 \times 15 \mathrm{~cm}^{2}$ cone. Central-axis depth dose characteristics for $7 \mathrm{MeV}, 9 \mathrm{MeV}$, and $9 \mathrm{MeV}$ with $1 \mathrm{~cm}$ bolus of $15 \times 15 \mathrm{~cm}^{2}$ cone. $X_{\text {con }}$ indicates $X$-ray contamination that is extracted from $R_{\mathrm{p}}$ plus $2 \mathrm{~cm}$

\begin{tabular}{lcccccccc}
\hline Nominal energy $(\mathrm{MeV})$ & Surface dose $(\%)$ & $d_{\max }(\mathrm{cm})$ & $R_{90}(\mathrm{~cm})$ & $R_{80}(\mathrm{~cm})$ & $R_{50}(\mathrm{~cm})$ & $R_{\mathrm{p}}(\mathrm{cm})$ & $E_{0}(\mathrm{MeV})$ & $X_{\mathrm{con}}(\%)$ \\
\hline 7 & 89.7 & 1.12 & 1.74 & 1.99 & 2.52 & 3.31 & 5.87 & 1.1 \\
9 & 80.5 & 2.03 & 2.72 & 2.98 & 3.55 & 4.38 & 8.27 & 1.1 \\
9 with bolus & 91.1 & 1.03 & 1.72 & 1.98 & 2.55 & 3.32 & 5.94 & - \\
\hline
\end{tabular}


Table 4 Output factors for $7 \mathrm{MeV}$ electron beam. Output factors for $7 \mathrm{MeV}$ electron beam (reference: measured output for $9 \mathrm{MeV}$ beam of $10 \times 10 \mathrm{~cm}^{2}$ cone)

\begin{tabular}{lccc}
\hline Cone $\left(\mathrm{cm}^{2}\right)$ & $\begin{array}{l}\text { Output factor } \\
\text { calculated }\end{array}$ & $\begin{array}{l}\text { Output factor } \\
\text { measured }\end{array}$ & Difference (\%) \\
\hline $10 \times 10$ & 0.888 & 0.882 & 0.6 \\
$15 \times 15$ & 0.980 & 0.972 & 0.8 \\
\hline
\end{tabular}

were fabricated to produce $7 \mathrm{MeV}$ electron beams. The relative central-axis depth-dose curves measured at $100 \mathrm{~cm}$ SSD are plotted in Fig. 5a and b along with the corresponding calculated values. The agreement between the calculations and measurements was found to be within $2-3 \%$ at $d_{\max }$ for the two cone sizes employed in this study. The disagreement was larger in the region closer to the phantom surface for both cones. Crossbeam dose profiles of the $7 \mathrm{MeV}$ beam at $d_{\max }$ are shown in Fig. $5 \mathrm{c}$ and $\mathrm{d}$ for $10 \times 10$ and $15 \times 15 \mathrm{~cm}^{2}$ cones, respectively. All the measured profiles were in agreement with the calculated cross-beam dose profiles within $2-3 \%$ except for the penumbra and beyond the penumbra.

As shown in Fig. 6, the central-axis depth dose characteristics were compared with those of the standard $6 \mathrm{MeV}$ beam and the $9 \mathrm{MeV}$ beams with and without a $1 \mathrm{~cm}$ bolus. The characteristics of the $9 \mathrm{MeV}$ beam with the bolus were similar to that of our $7 \mathrm{MeV}$ beam, but had a surface dose a few \% higher than the $7 \mathrm{MeV}$ beam. The $7 \mathrm{MeV}$ beam showed a reduced penetration to a depth between the 6 and $9 \mathrm{MeV}$ beams, as well as an increased skin dose up to approximately $90 \%$ of dose maximum. As shown in Tables 2 and 3, the practical range, $R_{\mathrm{p}}$, reduced from 4.39 to $3.35 \mathrm{~cm}$ for $10 \times 10 \mathrm{~cm}^{2}$ cone and from 4.38 to $3.31 \mathrm{~cm}$ for $15 \times 15 \mathrm{~cm}^{2}$ cone by using the $7 \mathrm{MeV}$ beam instead of the $9 \mathrm{MeV}$ beam. The values of $d_{\max }$ were $1.16 \mathrm{~cm}$ and $1.12 \mathrm{~cm}$ for the $10 \times 10$ and $15 \times 15 \mathrm{~cm}^{2}$ cones, respectively.

\section{Output factors}

The presence of the ED through the beamline caused a decrease in output due to the scattered electrons from the ED with large angles. Table 4 shows the calculated and measured output factors for the new $7 \mathrm{MeV}$ beams. The reference of the output factor was measured using an open $10 \times 10 \mathrm{~cm}^{2}$ cone of a $9 \mathrm{MeV}$ beam, which has a value of 1.0. The measured output factors of the $7 \mathrm{MeV}$ beam were 0.882 and 0.972 for $10 \times 10 \mathrm{~cm}^{2}$ and $15 \times 15 \mathrm{~cm}^{2}$, respectively. The measurements and calculations were within $1 \%$ of each other for the $10 \times 10$ and the $15 \times 15 \mathrm{~cm}^{2}$ cones. Table 5 shows the measured cutout factors of our $7 \mathrm{MeV}$ beam for several different sizes of circular electron beam blocks of $10 \times 10 \mathrm{~cm}^{2}$ and $15 \times 15 \mathrm{~cm}^{2}$ cones. The cutout factor of the $9 \mathrm{MeV}$ beam showed no significant variation, however the cutout factor of the $7 \mathrm{MeV}$ beam widely varied with sizes of cutout inserts.

\section{In-vivo dosimetry}

Table 6 summarizes the results of in-vivo dosimetry for three patients in different irradiation conditions. In Table 6, the differences are expressed as the measured dose relative to the expected surface dose (160 cGy) on the central axis. We assume that the expected value at the surface dose was $89 \%$ of the maximum dose. Differences between measured and expected doses were less than 8 cGy except in the case of one OSLD. The deviation of OSLD 4 could be explained by its large off-axis distance (approximately $4 \mathrm{~cm}$ ) and thus a prolonged SSD due to the curved skin.

\section{Discussion}

We developed energy degraders for a new electron energy for the breast boost irradiation when using the electron mode of a commercial LINAC. Iterative $\mathrm{MC}$ simulations were performed to obtain the optimal structure and dimensions of the EDs. By placing the ED in the lowermost scraper of the applicator to minimize scattered doses out of the treatment field, it was also secured during LINAC rotation without an extra fixing device. The design of EDs can be considered as one of the alterative of modifying the LINAC's own energy when we need certain electron beam between energies. Thus, the developed method can be easily applied to other intermediate energies by selecting the base design of this study which consists of a truncated cone attached on top of a plane plate.

A Lucite ${ }^{\circ}$ plate was shown to be a suitable material for the reduction of X-ray contamination. ${ }^{33}$ Scattered

Table 5 Measured cutout factors for $7 \mathrm{MeV}$ electron beam. Measured cutout factors for $7 \mathrm{MeV}$ electron beam (reference: measured output for $9 \mathrm{MeV}$ beam of $10 \times 10 \mathrm{~cm}^{2}$ cone; A/P: area to perimeter)

\begin{tabular}{|c|c|c|c|c|c|c|c|c|c|c|c|}
\hline \multirow[b]{2}{*}{ Energy } & \multirow[b]{2}{*}{ Cone size $\left(\mathrm{cm}^{2}\right)$} & \multicolumn{10}{|c|}{$\mathrm{A} / \mathrm{P}$ ratio } \\
\hline & & 0.75 & 1.00 & 1.25 & 1.50 & 1.75 & 2.00 & 2.25 & 2.50 & 3.00 & 3.75 \\
\hline \multirow[t]{2}{*}{$7 \mathrm{MeV}$} & $10 \times 10$ & 0.335 & 0.490 & 0.623 & 0.738 & 0.805 & 0.858 & 0.870 & 0.882 & - & - \\
\hline & $15 \times 15$ & - & - & - & - & - & 0.900 & 0.922 & 0.946 & 0.963 & 0.972 \\
\hline \multirow[t]{2}{*}{$9 \mathrm{MeV}$} & $10 \times 10$ & 0.903 & 0.952 & 0.983 & 1.000 & 1.005 & 1.008 & 1.004 & 1.000 & - & - \\
\hline & $15 \times 15$ & - & - & - & - & - & 1.003 & 1.005 & 1.007 & 1.004 & 0.996 \\
\hline
\end{tabular}


Table 6 In vivo dose results. In vivo doses of 7 MeV beam measured with optically stimulated luminescence dosimeters

\begin{tabular}{cllll}
\hline $\begin{array}{l}\text { OSLD } \\
\text { number }\end{array}$ & $\begin{array}{l}\text { SSD } \\
(\mathrm{cm})\end{array}$ & $\begin{array}{l}\text { Cone size } \\
\left(\mathrm{cm}^{2}\right)\end{array}$ & $\begin{array}{l}\text { Measure Dose } \\
(\mathrm{cGy})\end{array}$ \\
\hline Patient 1 & 1 & 100 & 10 & 156 \\
& 2 & & & 153 \\
Patient 2 & 3 & 100 & 15 & 152 \\
& 4 & & & 139 \\
Patient 3 & 5 & 105 & 15 & 152 \\
& 6 & & & 152 \\
\hline
\end{tabular}

electrons from the ED that interact with the applicator are able to cause X-ray contamination. Since the X-ray contamination of electron beams is produced mainly in the scattering foils of the LINAC [43, 44]. The slight increase $(0.1 \%)$ in the X-ray contamination of depth-dose curves of the $7 \mathrm{MeV}$ beams was most likely caused by the ED. The Lucite ${ }^{\oplus} \mathrm{ED}$ in place can be used to raise the surface dose of a $9 \mathrm{MeV}$ electron beam to up to $90 \%$ of dose maximum as well as to reduce the penetration depth of electrons. Thus, the technique suggested here could be an acceptable alternative to the use of a bolus for boost treatment of the tumour bed of the breast. In particular, the ED minimizes the variation in patient's setup. Also the setup time is reduced when the ED is implemented, as the device is located on the lowermost scraper of the electron applicator rather than on the patient's skin. As shown in Fig. 6, this technique is able to fill the energy gap between $6 \mathrm{MeV}$ and $9 \mathrm{MeV}$ to some degree.

The tantalum wire mesh bolus and metal bolus increased the surface dose and kept only a small change of the depth dose curve $[4,7]$. These materials should be placed on the patient surface. It may cause the setup variations during the treatment course. However, the final design of the ED is easily fixed to the lowermost scarper of electron applicator at the same position for every fraction as shown in Fig. 1a.

The results of the in-vivo dosimetry showed lower than the expected value on the central axis (see Table 6). The measured points on the skin were several $\mathrm{cm}$ away from the central axis as shown in Fig. 2. Since our EDs were designed to achieve optimal uniformity at $d_{\max }$, the cross-beam dose profile at shallow depth was somewhat parabolic from the center to the penumbra, rather than uniform. Furthermore, the measured skin doses could vary with off-axis distances due to varying SSDs caused by the slope of the breast. These reasons may contribute to the lower in-vivo results than the expected value. Nonetheless, all in-vivo doses to the skin ranged from 78 to $87 \%$ of the prescription dose.

Unfortunately, current treatment planning systems (TPS) do not provide adequate flexibility to support beam data from a modified electron spoiler or ED on the electron applicator. Thus, the 3D dose distribution with the EDs was not calculated by the TPS. An inhouse Monte Carlo-based TPS is under development to evaluate the 3D dose distribution of the $7 \mathrm{MeV}$ beam.

\section{Conclusions}

In this study we developed novel energy degraders for $7 \mathrm{MeV}$ electron beams that are capable of reducing the penetration depth and enhancing the skin dose, compared to the standard $9 \mathrm{MeV}$ beam. The efficacy of the optimally-designed EDs was validated through experimental evaluation. Thus the optimally designed ED in the $9 \mathrm{MeV}$ beamline provides breast conserving patients with a new energy option of $7 \mathrm{MeV}$ for boost of the shallow tumor bed. It would be an alternative to bolus and thus eliminate inconvenience and concern about the daily variation of bolus setup.

\section{Acknowledgements \\ The authors would like to thank Mr. Joel N.K Carlson for editing this manuscript.}

\section{Funding}

This work was supported by the National Research Foundation of Korea (NRF) grant funded by the Korean government (MSIP: Ministry of Science, ICT and Future Planning) (No. NRF-2013M2B2B1075776) and the Nuclear Safety Research Program through the Korea Foundation of Nuclear Safety (KoFONS), granted financial resource from the Nuclear Safety and Security Commission (NSSC), Republic of Korea (No. 1503002).

\section{Availability of data and materials}

The datasets supporting the conclusions of this article are included within the article.

\section{Authors' contributions}

JIP drafted the manuscript, performed experiments and simulations. SWH provided insights about the study concept, reviewed and monitored the in-vivo dosimetry during the measurements. JIK managed and commented on methods for experiments and simulations. HSL and JGL performed experiments. IHK gave clinical insights about the study. SJY conceived of the study concept, managed and commented on experiments and simulations, and participated in all aspect of its design and coordination and helped draft the manuscript. All authors read and approved the final manuscript.

\section{Competing interests}

The authors declare that they have no competing interests.

\section{Consent for publication}

Written consent for publication was obtained from the patient.

\section{Ethics approval and consent to participate}

All patients signed, at hospital admission, consent for in-vivo dosimetry and scientific investigation. All procedures followed were in accordance with the ethical standards; an approval by an ethics committee was not applicable.

\section{Author details}

${ }^{1}$ Department of Transdisciplinary Studies, Program in Biomedical Radiation Sciences, Seoul National University Graduate School of Convergence Science and Technology, Seoul 151-742, Korea. ${ }^{2}$ Institute of Radiation Medicine, Seoul National University Medical Research Center, Seoul, Korea. ${ }^{3}$ Department of Radiation Oncology, Seoul National University Hospital, Seoul, Korea.

${ }^{4}$ Interdisciplinary Program in Radiation Applied Life Science, Seoul National University College of Medicine, Seoul, Korea. ${ }^{5}$ Advanced Institutes of Convergence Technology, Seoul National University, Suwon, Korea. 
Received: 5 January 2016 Accepted: 19 August 2016

\section{Published online: 31 August 2016}

\section{References}

1. Hogstrom KR, Almond PR. Review of electron beam therapy physics. Phys Med Biol. 2006;51:R455-89.

2. Khan FM. The physics of radiation therapy. 4th ed. Philadelphia: Lippincott, Williams \& Wilkins; 2009.

3. Alasti H, Galbraith DM. Depth dose flattening of electron beams using a wire mesh bolus. Med Phys. 1995;22:1675-83.

4. Cederbaum M, Ravkin A, Rosenblatt E, Gez E. Implementing a tantalum wire mesh to increase the skin dose in low-energy electron irradiation of the chest wall. Med Dosim. 2001:26:275-9.

5. Lambert GD, Richmond ND, Kermode RH, Porter DJT. The use of high density metal foils to increase surface dose in low-energy clinical electron beams. Radiother Oncol. 1999;53:161-6.

6. Low DA, Starkschall G, Bujnowski SW, Wang LL, Hogstrom KR. Electron bolus design for radiotherapy treatment planning - bolus design algorithms. Med Phys. 1992;19:115-24.

7. Low DA, Starkschall G, Sherman NE, Bujnowski SW, Ewton JR, Hogstrom KR. Computer-aided design and fabrication of an electron bolus for treatment of the paraspinal muscles. Int J Radiat Oncol Biol Phys. 1995;33:1127-38.

8. Deng J, Lee MC, Ma CM. A Monte Carlo investigation of fluence profiles collimated by an electron specific MLC during beam delivery for modulated electron radiation therapy. Med Phys. 2002;29:2472-83.

9. Gauer T, Albers D, Cremers F, Harmansa R, Pellegrini R, Schmidt R. Design of a computer-controlled multileaf collimator for advanced electron radiotherapy. Phys Med Biol. 2006;51:5987-6003.

10. Gauer T, Sokoll J, Cremers F, Harmansa R, Luzzara M, Schmidt R. Characterization of an add-on multileaf collimator for electron beam therapy. Phys Med Biol. 2008;53:1071-85.

11. Hogstrom KR, Boyd RA, Antolak JA, Svatos MM, Faddegon BA, Rosenman JG. Dosimetry of a prototype retractable eMLC for fixed-beam electron therapy. Med Phys. 2004;31:443-62.

12. Lee MC, Jiang SB, Ma CM. Monte Carlo and experimental investigations of multileaf collimated electron beams for modulated electron radiation therapy. Med Phys. 2000;27:2708-18.

13. Ma CM, Pawlicki T, Lee MC, Jiang SB, Li JS, Deng J, et al. Energy- and intensity-modulated electron beams for radiotherapy. Phys Med Biol. 2000;45:2293-311.

14. Ravindran BP, Singh IR, Brindha S, Sathyan S. Manual multi-leaf collimator for electron beam shaping-a feasibility study. Phys Med Biol. 2002;47:4389-96.

15. Vatanen $\mathrm{T}$, Traneus $\mathrm{E}$, Lahtinen T. Dosimetric verification of a Monte Carlo electron beam model for an add-on eMLC. Phys Med Biol. 2008;53:391-404.

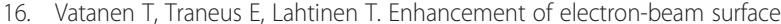
dose with an electron multi-leaf collimator (eMLC): a feasibility study. Phys Med Biol. 2009:54:2407-19.

17. du Plessis FCP, Leal A, Stathakis S, Xiong W, Ma CM. Characterization of megavoltage electron beams delivered through a photon multi-leaf collimator (pMLC). Phys Med Biol. 2006;51:2113-29.

18. Al-Yahya K, Verhaegen F, Seuntjens J. Design and dosimetry of a few leaf electron collimator for energy modulated electron therapy. Med Phys. 2007;34:4782-91.

19. Das IJ, Kase KR, Copeland JF, Fitzgerald TJ. Electron beam modifications for the treatment of superficial malignancies. Int J Radiat Oncol Biol Phys. 1991;21:1627-34

20. Rogers DW, Faddegon BA, Ding GX, Ma CM, We J, Mackie TR. BEAM: a Monte Carlo code to simulate radiotherapy treatment units. Med Phys. 1995;22:503-24.

21. Walters B, Kawrakow I, Rogers D: DOSXYZnrc users manual. NRC Report PIRS. 2005;794

22. Antolak JA, Bieda MR, Hogstrom KR. Using Monte Carlo methods to commission electron beams: a feasibility study. Med Phys. 2002;29:771-86.

23. Bieda MR, Antolak JA, Hogstrom KR. The effect of scattering foil parameters on electron-beam Monte Carlo calculations. Med Phys. 2001;28:2527-34.

24. Kapur A, Ma CM, Mok EC, Findley DO, Boyer AL. Monte Carlo calculations of electron beam output factors for a medical linear accelerator. Phys Med Biol. 1998:43:3479-94.

25. Ma CM, Jiang SB. Monte Carlo modelling of electron beams from medical accelerators. Phys Med Biol. 1999;44:R157-89.
26. Sheikh-Bagheri D, Rogers DW. Sensitivity of megavoltage photon beam Monte Carlo simulations to electron beam and other parameters. Med Phys. 2002:29:379-90

27. Verhaegen F, Mubata C, Pettingell J, Bidmead AM, Rosenberg I, Mockridge D, et al. Monte Carlo calculation of output factors for circular, rectangular, and square fields of electron accelerators (6-20 MeV). Med Phys. 2001;28:938-49.

28. Ye SJ, Pareek PN, Spencer S, Duan J, Brezovich IA. Monte Carlo techniques for scattering foil design and dosimetry in total skin electron irradiations. Med Phys. 2005;32:1460-8.

29. Park JM, Kim Jl, Heon Choi C, Chie EK, Kim IH, Ye SJ. Photon energy-modulated radiotherapy: Monte Carlo simulation and treatment planning study. Med Phys. 2012;39:1265-77

30. O'Shea TP, Sawkey DL, Foley MJ, Faddegon BA. Monte Carlo commissioning of clinical electron beams using large field measurements. Phys Med Biol. 2010:55:4083-105.

31. Ding GX, Rogers DWO. Energy spectra, angular spread and dose distribution of electron beam from various accelerators used in radiotherapy. National Research Council of Canada Report PIRS-0439. Ottawa: NRC; 1995

32. Zhang S, Liengsawangwong $P$, Lindsay $P$, Prado $K$, Sun TL, Steadham R, et al. Clinical implementation of electron energy changes of varian linear accelerators. J Appl Clin Med Phys. 2009;10:177-87.

33. ICRU. Radiation dosimetry: electron beams with energies between 1 and 50 MeV. ICRU Report. Bethesda: ICRU; 1984. p. 35

34. Almond PR, Biggs PJ, Coursey BM, Hanson WF, Huq MS, Nath R, et al. AAPM's TG-51 protocol for clinical reference dosimetry of high-energy photon and electron beams. Med Phys. 1999:26:1847-70.

35. Khan FM, Doppke KP, Hogstrom KR, Kutcher GJ, Nath R, Prasad SC, et al. Clinical electron-beam dosimetry: report of AAPM Radiation Therapy Committee Task Group No. 25. Med Phys. 1991;18:73-109.

36. Zhang GG, Rogers DWO, Cygler JE, Mackie TR. Monte Carlo investigation of electron beam output factors versus size of square cutout. Med Phys. 1999;26:743-50.

37. Chow JC, Grigorov GN. Electron radiotherapy: a study on dosimetric uncertainty using small cutouts. Phys Med Biol. 2007;52:N1-11.

38. Chen JZ, VanDyk J, Lewis C, Battista JJ. A two-source model for electron beams: calculation of relative output factors. Med Phys. 2001;28:1735-45.

39. Park SY, Ahn BS, Park JM, Ye SJ, Kim $H_{1}$, Kim Jl. Dosimetric comparison of $4 \mathrm{MeV}$ and $6 \mathrm{MeV}$ electron beams for total skin irradiation. Radiat Oncol. 2014;9:197.

40. Gibbons JP, Antolak JA, Followill DS, Huq MS, Klein EE, Lam KL, et al. Monitor unit calculations for external photon and electron beams: Report of the AAPM Therapy Physics Committee Task Group No. 71. Med Phys. 2014:41:031501.

41. Jursinic PA. Characterization of optically stimulated luminescent dosimeters, OSLDs, for clinical dosimetric measurements. Med Phys. 2007;34:4594-604.

42. Mills MD, Fajardo LC, Wilson DL, Jodi LD, William JS. Commissioning of a mobile electron accelerator for intraoperative radiotherapy. J Appl Clin Med Phys. 2001;2:121-30.

43. Eldib A, Jin L, Li J, Charlie Ma CM. Investigation of the clinical potential of scattering foil free electron beams. Phys Med Biol. 2014:59:819-36.

44. Connell T, Alexander A, Evans M, Seuntjens J. An experimental feasibility study on the use of scattering foil free beams for modulated electron radiotherapy. Phys Med Biol. 2012;57:3259-72.

\section{Submit your next manuscript to BioMed Central and we will help you at every step:}

- We accept pre-submission inquiries

- Our selector tool helps you to find the most relevant journal

- We provide round the clock customer support

- Convenient online submission

- Thorough peer review

- Inclusion in PubMed and all major indexing services

- Maximum visibility for your research

Submit your manuscript at www.biomedcentral.com/submit 